\title{
Promising Drug Design Strategies: Intrinsically Disordered Proteins
}

\author{
Michaux $\mathrm{C}^{1,2,3 *}$, Mignon J ${ }^{1}$ and Perpète EA ${ }^{1,2,4}$ \\ ${ }^{1}$ Laboratoire de Chimie Physique des Biomolécules, UCPTS, University of Namur, Belgium \\ ${ }^{2}$ Namur Institute of Structured Matter, University of Namur, Belgium \\ ${ }^{3}$ Namur Research Institute for Life Sciences, University of Namur, Belgium \\ ${ }^{4}$ Institute of Life-Earth-Environment, University of Namur, Belgium
}

\section{Mini Review}

Volume 3 Issue 2

Received Date: August 20, 2020

Published Date: September 15, 2020

DOI: $10.23880 /$ aabsc-16000152

*Corresponding author: Michaux C, Laboratoire de Chimie Physique des Biomolécules, Unité

de Chimie Physique Théorique et Structurale (UCPTS), University of Namur, Rue de Bruxelles, 61, 5000 Namur, Belgium, Tel: +32 81724557; E-mail: catherine.michaux@unamur.be

Keywords: Intrinsically disordered proteins; Therapeutic targets; Drug design; Protein-Protein Interactions

\section{Introduction}

In contrast to the classical paradigm "one sequence one structure - one function" that a given protein sequence corresponds to a well-defined three-dimensional (3D) structure and an associated function, it was discovered in the 1990s that an increasing number of proteins can be functional in the absence of a stable 3D-structure [1]. This new concept, the "disorder-function paradigm", assumes that an intrinsically flexible protein may have several structures and consequently various functions. Several terms were used to name these proteins: e.g. intrinsically disordered proteins (IDPs), natively unfolded proteins (NUPs), natively denatured proteins (NDPs) or intrinsically unstructured proteins (IUPs). Whereas some IDPs are predicted to be fully disordered, most of them are also known to have both structured domains and disordered regions.

Their native state is characterised as a dynamic ensemble of interconverting conformations under physiological conditions. The free energy landscape of IDPs can be described as a hilly plateau with numerous local energy minima, which is radically different from what is observed for ordered globular proteins, i.e. a well-defined global energy minimum [2]. This conformational plasticity is associated with specific sequence features including a low proportion of bulky hydrophobic amino acids and a high content of charged and hydrophilic residues [3-4]. Indeed, the combination of low mean hydrophobicity and high net charge is an important prerequisite for the absence of compact structure in a protein [5]. Depending on the environmental conditions and their binding partner, IDPs may exist in at least four conformations, the so-called protein quartet model: folded (ordered), molten globule, pre-molten globule, and random coil [6].

Disorder in proteins is highly prevalent in many organisms $[7,8]$. Indeed, more than one third of the eukaryotic proteins contain intrinsically disordered regions (IDRs) whereas for humans it is up to the half. Despite a lack of stable 3D-structure, IDPs play key roles in cellular processes, such as i) regulation of transcription, translation and cell cycle, ii) molecular recognition, and iii) chromatin organisation. Their functions usually operate by the binding of multiple protein partners [9]. Hence, most of IDPs are serving as hubs in protein-protein interactions (PPIs) networks $[10,11]$. IDPs are also regulated by extensive posttranslational modifications (PTMs), such as phosphorylation, acetylation and methylation, that affect their abundance, cellular distribution, fold ability and functions $[12,13]$.

IDPs are overrepresented amongst proteins implicated in disease [14-16]. More than 79\% of human cancer-associated proteins [17] and $57 \%$ of the identified cardiovascular disease-associated proteins [18] are predicted to contain intrinsically disordered regions. As they tend to misfold and aggregate under certain conditions, IDPs are associated with neurodegenerative disorders $[19,20]$ such as Alzheimer's or Parkinson's diseases. Whilst IDPs are essential therapeutic targets [21], only a small number of molecules and peptides are able to inhibit their functions. Often, the conventional structure-based drug design strategies applicable to ordered proteins are not appropriate for IDPs, due to their 
highly dynamic nature. Developing drugs targeting IDPs is therefore challenging. Currently, the most studied IDPs are: i) the nuclear protein NUPR1 [22] involved in pancreatic adenocarcinoma; ii) the transcription factor p53 [23] playing a role in cell cycle regulation, apoptosis and DNA repair; iii) the $\alpha$-synuclein protein [24] that enters in the so-called synucleinopathies, a group of neurodegenerative disorders; iv) the transcription factor and oncoprotein c-Myc [25] which is involved in a broad range of human cancers.

\section{IDPs as Druggable Targets}

Currently, four drug design strategies are implemented to target IDPs and disrupt their biological functions [26-30]. The first one consists in developing small molecules that bind to the IDP ordered domains, causing the disordered region to become ordered. It is called an allosteric inhibition [29].

In the second approach, drugs are able to mimic the ordered (or disordered) binding partner of the IDP preventing its binding and consequently the associated PPIs. Several small molecules have already shown abilities to inhibit the disordered interface of PPIs based on the disordered regions [7]. For instance, by using the molecular recognition features (MoRFs) method [31], the p53-Mdm2 interactions [28] can be targeted by peptides or small molecules [26].

The third strategy is more challenging and considers blocking IDPs' disordered regions by stabilising the functionally misfolded structure. It allows for a conformational transition from a disordered to an ordered structure. That is, the ligand has to directly block all the IDP disordered states. Few rational drug design methods have been proposed in this context. A computational approach has recently been described to virtually screen compounds that can simultaneously bind to different conformations of the oncoprotein c-Myc. The latter contains a disordered basic helix-loop-helix-leucine zipper domain that adopts a helical conformation upon binding to Myc-associated factor X (Max) [27]. Furthermore, a methodology based on the synergy of biophysical, computational and biological methods have been set up to identify a drug against NUPR1 [32].

A fourth method is to prevent aggregation of IDPs, which form toxic amyloid oligomers or fibrils [30]. Several small molecules have been reported to interfere with the aggregation process of the $A \beta$ peptide, an IDP associated with Alzheimer's disease [33], or the $\alpha$-synuclein [34] involved in Parkinson's pathology.

\section{Biophysical Characterisation of IDPs}

In order to understand the IDPs functional mechanism and promote novel strategies for drug discovery, it is essential to first investigate their structural characteristics and their specific interactions with binding partners in their PPIs network. In this context, combining biophysical and computational methods turned out to be the most relevant strategy. Indeed, a serious bottleneck for this type of analyses is that no single method can provide access to the inherent flexibility and structural variability of IDPs; traditional structural biology method such as X-ray crystallography is not adapted.

Alternative techniques such as nuclear magnetic resonance (NMR), small-angle X-ray scattering (SAXS), circular dichroism (CD) or single-molecule fluorescence (particularly, the Förster resonance energy transfer method) can be used to attempt IDPs structural characterisation [35]. NMR provides local and long-range structural information while SAXS measures protein compactness and shape. Residual secondary structures and IDRs are revealed by $\mathrm{CD}$, and fluorescence gives information about the structural heterogeneity and the intermediate structures.

Guided by experimental data, computational methods [36] may also help interpreting the results at the atomic scale and giving information about individual conformations in the ensemble. Molecular dynamics-based methods [37] are the most used, showing the atomic fluctuations of IDPs as a function of time. However, they still suffer some weaknesses such as the selection of an appropriate force-field or the need to generate uncorrelated atomistic microstructures. Several strategies are proposed to overcome these problems and produce a correct description of the IDP conformational ensemble $[38,39]$.

\section{Perspective}

IDPs constitute a unique class of proteins with crucial biological functions. In recent years, multiple studies have highlighted their intrinsic properties and binding mechanisms. However, more efforts are still needed to fully understand these fuzzy systems and particularly the PPIs in which they are involved. Such insights are precious for the rational design of therapy targeting IDPs, which are present in human diseases.

\section{Acknowledgments}

C.M. and E.A.P. thank the Belgian National Fund for Scientific Research for their Research Associate and Senior Research Associate positions, respectively.

\section{References}

1. Wright PE, Dyson HJ (1999) Intrinsically unstructured proteins: re-assessing the protein structure-function 
paradigm. Journal of Molecular Biology 293(2): 321-331.

2. Uversky VN (2016) Paradoxes and wonders of intrinsic disorder: Complexity of simplicity. Intrinsically Disordered Proteins 4(1): e1135015.

3. Uversky VN, Gillespie JR, Fink AL (2000) Why are "natively unfolded" proteins unstructured under physiologic conditions? Proteins 41(3): 415-427.

4. Lieutaud P, Ferron F, Uversky AV, Lukasz Kurgan, Vladimir N Uversky, et al. (2016) How disordered is my protein and what is its disorder for? A guide through the "dark side" of the protein universe. Intrinsically Disordered Proteins 4(1): e1259708.

5. Uversky VN (2011) Intrinsically disordered proteins from A to Z. International Journal of Biochemistry \& Cell Biology 43(8): 1090-1103.

6. Uversky VN (2002) Natively unfolded proteins: a point where biology waits for physics. Protein Science 11(4): 739-756.

7. Dunker AK, Oldfield CJ, Meng J, Pedro Romero, Jack Y Yang, et al. (2008) The unfoldomics decade: an update on intrinsically disordered proteins. BMC Genomics 9(2): S1.

8. Ward JJ, Sodhi JS, McGuffin LJ, Buxton BF, Jones DT (2004) Prediction and functional analysis of native disorder in proteins from the three kingdoms of life. Journal of Molecular Biology 337(3): 635-645.

9. Dyson HJ, Wright PE (2005) Intrinsically unstructured proteins and their functions. Nature Reviews Molecular Cell Biology 6(3): 197-208.

10. Perovic V, Sumonja N, Marsh LA, Sandro Radovanovic, Milan Vukicevic, et al. (2018) IDPpi: Protein-Protein Interaction Analyses of Human Intrinsically Disordered Proteins. Scientific Reports 8(1): 10563.

11. Singh GP, Ganapathi M, Dash D (2007) Role of intrinsic disorder in transient interactions of hub proteins. Proteins 66(4): 761-765.

12. Pejaver V, Hsu WL, Xin F, A Keith Dunker, Vladimir N Uversky, et al. (2014) The structural and functional signatures of proteins that undergo multiple events of post-translational modification. Protein Science 23(8): 1077-1093.

13. Darling AL, Uversky VN (2018) Intrinsic Disorder and Posttranslational Modifications: The Darker Side of the Biological Dark Matter. Frontiers in Genetics 9: 158.
14. Uversky VN, Oldfield CJ, Dunker AK (2008) Intrinsically disordered proteins in human diseases: introducing the D2 concept. Annual Reviews in Biophysics 37: 215-246.

15. Babu MM, van der Lee R, de Groot NS, Jörg Gsponer (2011) Intrinsically disordered proteins: regulation and disease. Current Opinion in Structural Biology 21(3): 432-440.

16. Uversky VN, Davé V, Iakoucheva LM, Prerna Malaney, Steven J Metallo, et al. (2014) Pathological unfoldomics of uncontrolled chaos: intrinsically disordered proteins and human diseases. Chemical Reviews 114(13): 68446879.

17. Iakoucheva LM, Brown CJ, Lawson JD, Zoran Obradović, A Keith Dunker (2002) Intrinsic disorder in cell-signaling and cancer-associated proteins. Journal of Molecular Biology 323(3): 573-584

18. Cheng Y, LeGall T, Oldfield CJ, Keith Dunker A, Vladimir $\mathrm{N}$ Uversky (2006) Abundance of intrinsic disorder in protein associated with cardiovascular disease. Biochemistry 45(35): 10448-10460

19. Uversky VN (2015) Intrinsically disordered proteins and their (disordered) proteomes in neurodegenerative disorders. Frontiers in Aging Neurosciences 7: 18.

20. Uversky VN (2014) The triple power of $D(3)$ : protein intrinsic disorder in degenerative diseases. Frontiers in Bioscience (Landmark Ed.) 19: 181-258.

21. Metallo SJ (2010) Intrinsically disordered proteins are potential drug targets. Current Opinion in Chemical Biology 14(4): 481-488.

22. Neira JL, López MB, Sevilla P, Bruno Rizzuti, Ana CámaraArtigas, et al. (2018) The chromatin nuclear protein NUPR1L is intrinsically disordered and binds to the same proteins as its paralogue. Biochemical Journal 475(14): 2271-2291.

23. Uversky VN (2016) p53 Proteoforms and Intrinsic Disorder: An Illustration of the Protein StructureFunction Continuum Concept. International Journal of Molecular Sciences 17(11): 1874.

24. Uversky VN, Eliezer D (2009) Biophysics of Parkinson's disease: structure and aggregation of alpha-synuclein. Current Protein \& Peptide Science 10(5): 483-499

25. Beaulieu ME, Castillo F, Soucek L (2020) Structural and Biophysical Insights into the Function of the Intrinsically Disordered Myc Oncoprotein. Cells 9(4): 1038.

26. Wang J, Cao Z, Zhao L, Shuqiang Li (2011) Novel strategies 
for drug discovery based on Intrinsically Disordered Proteins (IDPs). International Journal of Molecular Sciences 12(5): 3205-3219.

27. Yu C, Niu X, Jin F, Zhirong Liu, Changwen Jin, et al. (2016) Structure-based Inhibitor Design for the Intrinsically Disordered Protein c-Myc. Scientific Reports 6: 22298.

28. Cheng Y, LeGall T, Oldfield CJ, Mueller JP, Van YY, et al. (2006) Rational drug design via intrinsically disordered protein. Trends in Biotechnology 24(10): 435-442.

29. Joshi P,Vendruscolo M (2015) Druggability of Intrinsically Disordered Proteins. Advances in Experimental Medicine and Biology 870: 383-400.

30. Uversky VN (2012) Intrinsically disordered proteins and novel strategies for drug discovery. Expert Opinion on Drug Discovery 7(6): 475-488.

31. Van der Lee R, Buljan M, Lang B, Robert J Weatheritt, Gary W Daughdrill, et al. (2014) Classification of intrinsically disordered regions and proteins. Chem Rev 114(13): 6589-6631

32. Neira JL, Bintz J, Arruebo M, Bruno Rizzuti, Thomas Bonacci, et al. (2017) Identification of a Drug Targeting an Intrinsically Disordered Protein Involved in Pancreatic Adenocarcinoma. Scientific Reports 7: 39732.

33. Wang Q, Liang G, Zhang M, Jun Zhao, Kunal Patel, et al. (2014) De novo design of self-assembled hexapeptides as $\beta$-Amyloid $(A \beta)$ peptide inhibitors. ACS Chem Neurosci 5(10): 972-981.
34. Toth G, Gardai SJ, Zago W, Carlos W Bertoncini, Nunilo Cremades, et al. (2014) Targeting the intrinsically disordered structural ensemble of $\alpha$-synuclein by small molecules as a potential therapeutic strategy for Parkinson's disease. PloS ONE 9(2): e87133.

35. Uversky VN (2015) Biophysical Methods to Investigate Intrinsically Disordered Proteins: Avoiding an "Elephant and Blind Men" Situation. Advances in Experimental Medicine and Biology 870: 215-260.

36. Bhattacharya S, Lin X (2019) Recent Advances in Computational Protocols Addressing Intrinsically Disordered Proteins. Biomolecules 9(4): 146.

37. Kasahara K, Terazawa H, Takahashi T, Junichi Higo (2019) Studies on Molecular Dynamics of Intrinsically Disordered Proteins and Their Fuzzy Complexes: A MiniReview. Computational and Structural Biotechnology Journal 17: 712-720.

38. Curcó D, Michaux C, Roussel G, Tinti Emmanuel, Alemán C, et al. (2012) Stochastic simulation of structural properties of natively unfolded and denatured proteins. Journal of Molecular Modeling 18(9): 4503-4516.

39. ShresthaUR,JunejaP,ZhangQ,Viswanathan Gurumoorthy, Jose M Borreguero, et al. (2019) Generation of the configurational ensemble of an intrinsically disordered protein from unbiased molecular dynamics simulation. Proceedings of National Academy of Sciences 116(41): 20446-20452. 